Version: SePtember 6, 2021

Preprint typeset using $\mathrm{LAT}_{\mathrm{E}} \mathrm{X}$ style emulateapj v. 08/22/09

\title{
V1647 ORIONIS: REINVIGORATED ACCRETION AND THE RE-APPEARANCE OF MCNEIL'S NEBULA
}

\author{
Colin Aspin ${ }^{1}$, Bo Reipurth ${ }^{1}$, Tracy L. Beck ${ }^{2}$, Greg Aldering $^{3}$, Ryan L. Doering ${ }^{4}$ Heidi B. Hammel $^{5,9}$, \\ David K. LynCh $^{6,9}$, Margaret Meixner ${ }^{2}$ Emmanuel Pecontal $^{7}$, Ray W. Russell ${ }^{6,9}$, Michael L. Sitko ${ }^{8,9}$, \\ Rollin C. ThOMAS ${ }^{3}$, Vivian U ${ }^{1}$ \\ Version: September 6, 2021
}

\begin{abstract}
In late 2003, the young eruptive variable star V1647 Orionis optically brightened by over 5 magnitudes, stayed bright for around 26 months, and then decline to its pre-outburst level. In August 2008 the star was reported to have unexpectedly brightened yet again and we herein present the first detailed observations of this new outburst. Photometrically, the star is now as bright as it ever was following the 2003 eruption. Spectroscopically, a pronounced P Cygni profile is again seen in $\mathrm{H} \alpha$ with an absorption trough extending to $-700 \mathrm{~km} \mathrm{~s}^{-1}$. In the near-infrared, the spectrum now possesses very weak $\mathrm{CO}$ overtone bandhead absorption in contrast to the strong bandhead emission seen soon after the 2003 event. Water vapor absorption is also much stronger than previously seen. We discuss the current outburst below and relate it to the earlier event.
\end{abstract}

Subject headings: stars: individual (V1647 Ori) — circumstellar matter — stars: formation

\section{INTRODUCTION}

When V1647 Orionis went into outburst in the fall of 2003, it brightly illuminated a monopolar reflection nebula subsequently designated McNeil's Nebula (McNeil 2004). The young star itself increased in optical and near-IR (NIR, K-band) brightness by around 5 and 3 magnitudes, respectively, and sparked a world-wide effort to study the details of this rare type of eruption. To date, this has resulted in over 50 research papers which relate the characteristics of the star and nebula from the time of its detection to the time when it had faded to its pre-outburst optical brightness (in February 2006). The reader is referred to the most recent publication on V1647 Ori, (Aspin, Beck, \& Reipurth 2008, ABR08), for a comprehensive list of previous work.

It has been speculated that V1647 Ori may be a new example of the class of young variable stars designated "FUor" after the class progenitor, FU Orionis (Ambartsumian 1971). Only about ten so-called "classical" FUors, (those that have been directly observed to optically brighten over a period of a few weeks to a few months) have so far been found. A comprehensive review of the properties of FUors was given by Hartmann \& Kenyon (1996), however, it is important to relate here the main characteristics defining this group. i) an ob-

\footnotetext{
${ }^{1}$ Institute for Astronomy, University of Hawaii, 640 N. A'ohoku Place, Hilo, HI 96720. E-mail: caa@ifa.hawaii.edu

2 Space Telescope Science Institute, 3700 San Martin Drive, Baltimore, MD 21218.

${ }^{3}$ Lawrence Berkeley Lab, Physics Div., MS-50/232, One Cyclotron Rd., Berkeley, CA 94720, USA

${ }^{4}$ Department of Physics and Astronomy, Valparaiso University, Valparaiso, IN 46383.

5 Space Science Institute, 4750 Walnut Street, Suite 205, Boulder, CO 80301.

${ }^{6}$ The Aerospace Corporation, Los Angeles, CA 90009.

7 Observatoire de Lyon, 9 Avenue Charles-Andre, 69561 SaintGenis-Laval Cedex, France

8 Department of Physics, University of Cincinnati, Cincinnati $\mathrm{OH} 45221$.

${ }^{9}$ Visiting Astronomer, NASA Infrared Telescope Facility, operated by the University of Hawaii under contract with the National Aeronautics and Space Administration.
}

served rise in optical brightness of around 5-6 magnitudes, ii) an optical spectrum indicative of a G- to F-type supergiant star, iii) a NIR K-band spectrum typical of a M-type giant, iv) the star remains in an elevated state for many years, even decades - FU Ori has declined little in over the 70 years since its discovery (Herbig (1966, 1977), v) an $\mathrm{H} \alpha$ line in emission with an associated blueshifted absorption component (i.e. a P Cygni profile), and vi) an associated, generally curving, reflection nebula. What has been gleamed with regard to V1647 Ori, however, is somewhat contradictory to these definitive characteristics in that it had a relatively short outburst period (approximately 26 months), its optical and NIR outburst spectra possessed strong atomic and molecular emission features, and the eruption was shown to be repetitive - Aspin et al. (2006) described an earlier event commencing in 1966. Such properties have more in common with those observed in the "EXor" class of eruptive variables, named after their class progenitor, EX Lupi (Herbig 1989).

It was expected that the next eruption of V1647 Ori would be $\sim 37$ years after the 2003 event, assuming the timescale between the 1966 and 2003 events was typical. However, in late-August 2008, some 18 months after the fading of the 2003 outburst, V1647 Ori was observed to brighten by around 5 magnitudes yet again and McNeil's Nebula became brightly visible (Itagaki et al. 2008). This was confirmed by Aspin (2008) who showed that V1647 Ori had brightened in the red optical $(\sim 6500 \AA)$ from r'=23.3 in March 2006 to $R=17.3$ in late-August 2008. Here we present new optical and NIR observations of V1647 Ori from late-August to early-September 2008. In $\S 2$ we briefly describe the observations and data reduction. In $\S 3$ we present the results, while in $\S 4$ we discuss the current event in relation to the 2003 outburst.

\section{OBSERVATIONS AND DATA REDUCTION}

Below we present new data taken on four different telescopes and with five different instruments. Optical Rband images (see Fig. 1) were obtained on the University of Hawaii (UH) 2.2 meter telescope on UT 2008 Septem- 

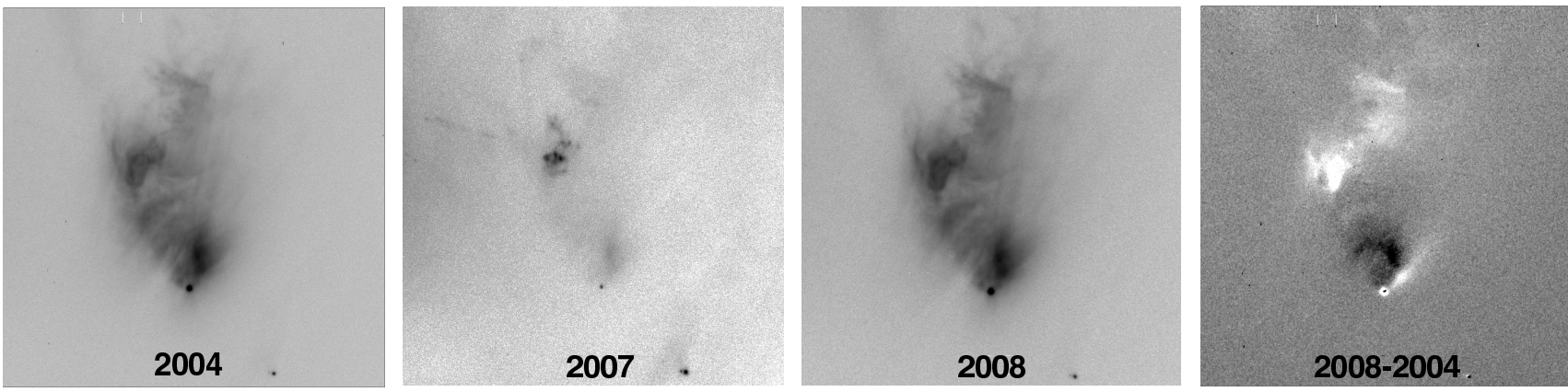

Fig. 1.- The region immediately surrounding V1647 Orionis and McNeil's Nebula at three different epochs. The left image was taken in an SDSS r' filter in 2004, around one year after the 2003 outburst. The middle-left image was taken in a standard Johnson R filter in 2007, around 10 months after V1647 Ori had returned to its pre-outburst optical brightness. The middle-right image was taken in the same R filter in 2008, soon after the recent brightening of the star and the re-appearance of McNeil' Nebula. The right image is a difference image, 2008-2004. In this image, white is more flux in 2008, black is more flux in 2004. We note that V1647 Orionis is the point-like object at the apex of the monopolar reflection nebula extending northward. The images were scaled to result in the cancellation of the faint binary star at the bottom of the image.

ber 1 using the Tektronix $2 \mathrm{~K} \times 2 \mathrm{~K}$ CCD camera (Tek2K). The on-source exposure time was 120 seconds and seeing was $\sim 0$.' 7 . The images were calibrated by using a sequence of six field stars described in Aspin, Herbig, \& Reipurth (2009, henceforth AHR09).

Optical images were also obtained on Gemini-South using GMOS (Davies et al. 1997; Hook et al. 2004) on UT 2008 September 22. The SDSS g', r', i', and z' filters were used and the on-source exposure times were 240, 120, 120, and 80 seconds, respectively. Details of the photometric calibration, can be found AHR09. Seeing during the observations was $\sim 00^{\prime \prime} 6$.

Optical spectroscopy was obtained on the UH 2.2 meter telescope on UT 2008 August 31 using the dualchannel integral-field unit (IFU) spectrograph, SNIFS (Aldering et al. 2002; Lantz et al. 2004). The $6^{\prime \prime} \times 6^{\prime \prime}$ field-of-view of the SNIFS spectrograph is filled with a $15 \times 15$ grid of lenslet-defined spatial elements, each sampling $\sim 0$ ". 4 . The data was reduced by the SNIFS reduction pipeline (Aldering et al. 2006). The spectral resolution of the final spectrum was $\mathrm{R} \sim 1850$ which was extracted from the IFU cube using a wavelength-dependent PSF fitting procedure (Aldering et al. 2006). Wavelength calibration was performed using the SNIFS internal calibration unit and flux calibration was performed using observations of spectrophotometric standards.

An optical spectrum was also obtained on GeminiSouth using GMOS on UT September 22. We used the R400 grating and a 0.'75 slit giving an effective spectral resolution of $\mathrm{R} \sim 1900$. Data was taken in nod-and-shuffle mode to aid in the removal of the bright red optical sky emission lines.

NIR spectroscopy was obtained on the NASA IRTF telescope on UT 2008 September 4 using the facility NIR spectrograph SpeX (Rayner et al. 2003). The crossdispersed (XD) mode was used and observations were acquired in both the short- and long-XD settings. The data were reduced using the SpeX IDL package (Cushing, Vacca, \& Rayner 2004). Telluric correction and flux calibration were performed using the $\mathrm{A} 0 \mathrm{~V}$ telluric standard star HD 39985. Conditions were clear and the seeing stable during the target and standard observations and hence we consider the flux calibration good to the $10 \%$ level.

Mid-IR (MIR) imaging was obtained on Gemini-South on UT September 13, 2008 with T-ReCS (Telesco et al. 1998) using broad $\mathrm{N}^{\prime}\left(\lambda_{c}=11.2 \mu \mathrm{m}\right)$ and narrow Qa $\left(\lambda_{c}=18.30 \mu \mathrm{m}\right)$ filters. Conditions were clear, dry and stable throughout the observations and photometric calibration was performed using similar observations of the bright "Cohen" standard star (Cohen et al. 1999) HD39400 ( $\left.\mathrm{N}^{\prime}=1.534, \mathrm{Qa}=1.434\right)$.

Near-IR images were obtained on the WIYN 3.5-meter telescope on UT September 16 2008. The WHIRC NIR camera (Meixner et al. 2008) was used with standard J, $\mathrm{H}$, and Ks filters. Flux calibration was achieved using observations of the UKIRT faint standard star FS 07 (Hunt et al. 1998).

\section{RESULTS}

In Fig. 1 we show a comparison of red optical images of V1647 Ori from three different epochs. The left image is from UT 2004 September 3 and was taken with an SDSS r' filter using GMOS. This was around 10-11 months after the 2003 outburst of V1647 Ori was first detected (McNeil 2004) and V1647 Ori had a magnitude of $\mathrm{r}^{\prime}=17.8$. The middle-left image shows the same region of sky but was taken on UT 2006 December 22 using a Johnson R filter with the UH Tek2K camera. At the time of these observations, V1647 Ori had been at its preoutburst (red optical) brightness of r' $=23.3$ for around 10 months. The middle-right is our recent UH Tek2K image detailed in $\S 2$ above. In this image, V1647 Ori has an optical brightness of $\mathrm{R}=17.3$. Comparing the left and middle-right images, we note that in both V1647 Ori and its associated nebula are strongly visible. However, in the middle-left image both the star and the nebula are only faintly visible. The bright knot visible in the middle-left image (top and left of center) is the Herbig-Haro object, HH 22. We note that the optical brightness of V1647 Ori in the 2004 and 2008 images is similar as is the brightness and morphology of McNeil's Nebula. The far-right image shows the difference between the 2004 and 2008 images with white implying that the object was brighter in 2008, and black meaning brighter in 2004. The images were scaled prior to subtraction to give the same signal in the faint binary system near the bottom (and left of center) of the images. Clearly, there are significant differences in brightness between the two epochs suggesting that in 2008, the reflection nebula is illuminated in a somewhat different manner than in 2004. This suggests that dust obscuration close to V1647 Ori may play a im- 

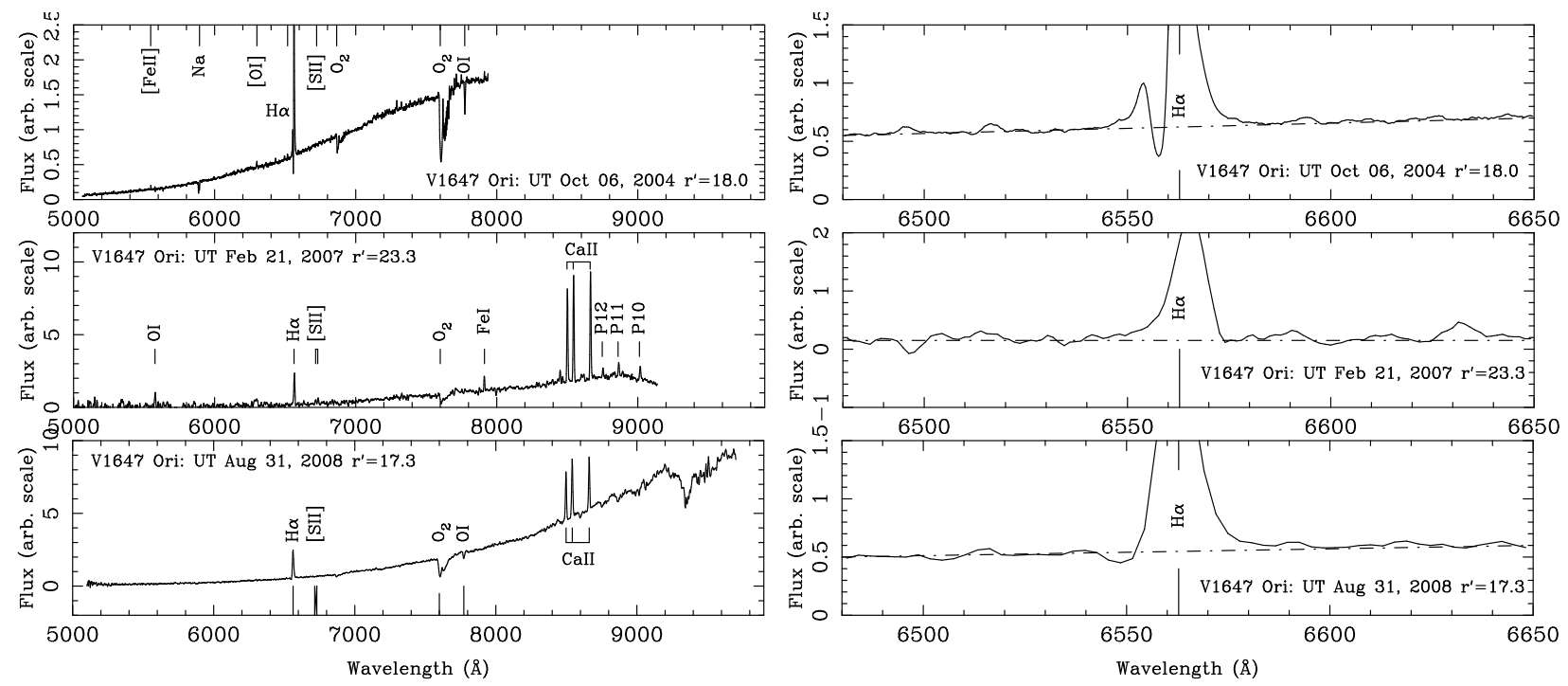

FIG. 2.- Optical spectra of V1647 Ori from three epochs, UT August 31, 2008, February 21, 2007, and October 6, 2004. The 2008 spectrum is from the latest outburst of the star while the 2004 spectrum was taken during the 2003-2006 event. The 2007 spectrum was taken around one year after V1647 Ori had returned to its pre-outburst optical brightness after the 2003 eruption had subsided. Note the 2004 spectrum did not include the Ca II lines. The left three panels show the full spectra range observed while the right three panels focus on the region immediately around the $\mathrm{H} \alpha$ emission feature.

portant role in defining the observed morphology of the nebula.

Photometric observations of V1647 Ori from the optical through mid-IR are shown in Table 1. Optical and NIR colors are also given. Within the associated uncertainities, the optical through NIR brightness values for V1647 Ori are comparable to those measured in February 2004 when the source was last observed to have brightened significantly (Reipurth \& Aspin 2004).

We present our new optical spectrum of V1647 Ori in Fig. 2 (bottom). We additionally show similar spectra from October 2004 (top) and February 2007 (middle) for direct comparison. In October 2004, V1647 Ori was some 11-12 months into its eruption and had not yet started to decline in optical brightness. In February 2007, the star had been at its pre-outburst optical brightness for around 1 year. In all three spectra, $\mathrm{H} \alpha$ is in emission although only in October 2004 and August 2008 did it show a blue-shifted absorption component (a P Cygni profile). On both these dates, the blueshifted $\mathrm{H} \alpha$ absorption extended to approximately $-700 \mathrm{~km} \mathrm{~s}^{-1}$ although in August 2008 the depth of the absorption is less than in October 2004. The full-width half maximum of the $\mathrm{H} \alpha$ emission is large with the line wings extending to around $\pm 1000 \mathrm{~km} \mathrm{~s}^{-1}$. This is somewhat larger than observed at the start of the 2004 event (i.e. $\sim 750 \mathrm{~km} \mathrm{~s}^{-1}$, see AHR09 for more details) suggesting the physical conditions in the $\mathrm{H}$ emission region are somewhat more extreme. Also present in the August 2008 spectrum are the Ca II triplet lines in emission. The ratios of these lines (1.6:2:1.7) are similar to those found in February 2004 (1.5:2:1.7) implying that unlike the emission seen in February 2007 (with ratios 1.7:2:1.9), conditions in the Ca II emission region have become optically thick producing saturated line emission. The Ca II emission lines are discussed in more detail in ABR08. Also present in our recent spectrum are O I $(6300 \AA)$ in emission, and O I $(7773 \AA)$ in absorption. Other weak features may be present but, with the spectral resolution obtained with SNIFS, it is
TABLE 1

Photometry of V1647 Ori

\begin{tabular}{lccc}
\hline \hline $\begin{array}{c}\text { Filter }(\lambda) \\
(\mu \mathrm{m})\end{array}$ & UT & $\begin{array}{c}2008 \text { Flux }^{\mathrm{a}} \\
(\text { mags })\end{array}$ & $\begin{array}{c}2004 \text { Flux } \\
(\mathrm{mags})\end{array}$ \\
\hline g' $(0.475)$ & $2008 / 09 / 22$ & $19.86 \pm 0.12$ & $20.05 \pm 0.15$ \\
V (0.55) & $2008 / 08 / 31$ & $18.10 \pm 0.15$ & $18.50 \pm 0.15^{\mathrm{c}}$ \\
r' $(0.63)$ & $2008 / 09 / 22$ & $17.53 \pm 0.07$ & $17.70 \pm 0.09$ \\
i' $(0.78)$ & $2008 / 09 / 22$ & $16.03 \pm 0.06$ & $15.90 \pm 0.08$ \\
z'(0.925) & $2008 / 09 / 22$ & $14.43 \pm 0.06$ & $14.39 \pm 0.06$ \\
J (1.25) & $2008 / 09 / 16$ & $10.62 \pm 0.07$ & $10.85 \pm 0.10^{\mathrm{c}}$ \\
H (1.65) & $2008 / 09 / 16$ & $8.90 \pm 0.07$ & $8.90 \pm 0.10^{\mathrm{c}}$ \\
Ks (2.2) & $2008 / 09 / 16$ & $7.53 \pm 0.07$ & $7.60 \pm 0.10^{\mathrm{c}}$ \\
N'(11.2) & $2008 / 09 / 13$ & $2.51 \pm 0.20$ & $1.50 \pm 0.20$ \\
Qa (18.3) & $2008 / 09 / 13$ & $0.62 \pm 0.20$ & - \\
g'-r' & $2008 / 09 / 22$ & $2.33 \pm 0.17$ & $2.35 \pm 0.12$ \\
r'-i' & $2008 / 09 / 22$ & $1.50 \pm 0.10$ & $1.80 \pm 0.09$ \\
r'-z' & $2008 / 09 / 22$ & $1.60 \pm 0.10$ & $1.51 \pm 0.08$ \\
J-H & $2008 / 09 / 22$ & $1.72 \pm 0.10$ & $1.95 \pm 0.14$ \\
H-K & $2008 / 09 / 22$ & $1.37 \pm 0.10$ & $1.30 \pm 0.14$ \\
& & & \\
\hline
\end{tabular}

${ }^{a}$ Data acquired during the current outburst.

b Data acquired in Feb-Mar 2004.

c Data from McGehee et al. (2004).

difficult to determine if they are real. Included in Table 2 are details of the optical spectral features present. Two lines that are conspicuously absent are the [S II] lines at 6717 and $6731 \AA$ which were present in February 2007. This is likely due to the already weak lines (see ABR08) being masked by the addition of strong continuum (accretion) emission which was not present in the February 2007 spectrum. Finally, we note that the SNIFS acquisition image of V1647 Ori gave a magnitude of V=18.1.

Fig. 3 shows the SpeX NIR spectrum of V1647 Ori (bottom). As in the optical, we also show spectra from two other epochs, in this case, February 2007 (middle) and December 2004 (top). Present in the August 2008 NIR spectrum are the atomic emission lines of He I (1.083 $\mu \mathrm{m}, \mathrm{P}$ Cygni profile), $\mathrm{Pa} \beta(1.282 \mu \mathrm{m})$, weak [Fe II] $(1.644 \mu \mathrm{m})$, He I $(2.058 \mu \mathrm{m}$, P Cygni profile), $\operatorname{Br} \gamma(2.166 \mu \mathrm{m})$, and $\operatorname{Br} \alpha(4.051 \mu \mathrm{m})$. Details of these 

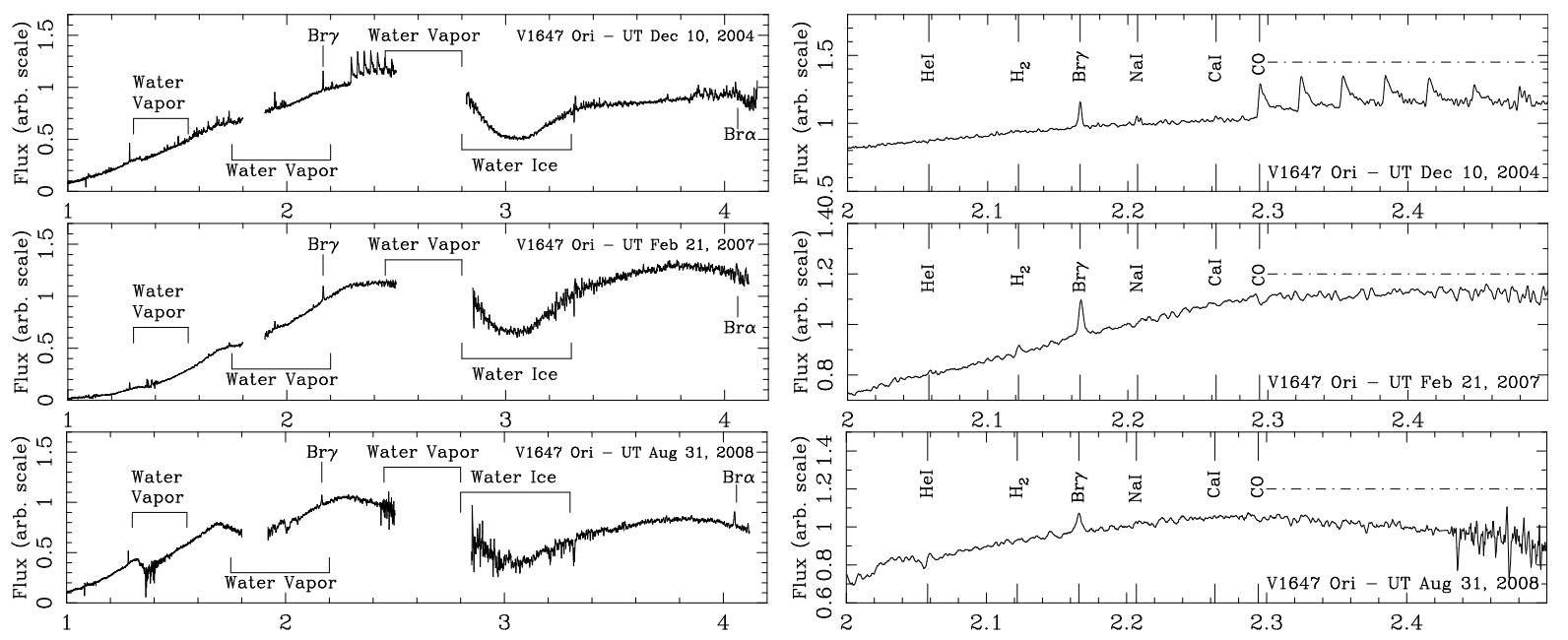

FIG. 3.- The NIR 1 to $4.2 \mu \mathrm{m}$ SpeX spectrum of V1647 Ori taken on UT August 31, 2008 (bottom panels). For comparison, we also show the same wavelength range from SpeX spectra from UT February 21, 2007 (middle panels) and UT December 10, 2004 (top panels). The main spectral features are identified. Features present are water vapor absorption bands (in J, H, and K), water ice absorption (at $3 \mu \mathrm{m}$ ), and emission lines of $\mathrm{Pa} \beta$ (at $1.282 \mu \mathrm{m}$ ), weak [Fe II] (at $1.644 \mu \mathrm{m})$, He I (at $2.058 \mu \mathrm{m}), \operatorname{Br} \gamma($ at $2.166 \mu \mathrm{m})$, and $\mathrm{Br} \alpha($ at $4.051 \mu \mathrm{m})$. The right three panels zoom into the K-band region of the three spectra. Note the CO bandheads (at 2.294 $\mu \mathrm{m}$ ) are in emission in 2004, are weakly in absorption in 2007, and perhaps very weakly in absorption in 2008.

TABLE 2

Spectral Features

\begin{tabular}{|c|c|c|c|c|}
\hline $\begin{array}{c}\lambda \\
(\mu \mathrm{m})\end{array}$ & $\begin{array}{l}\text { Line } \\
\text { ID }\end{array}$ & $\begin{array}{l}\mathrm{W}_{\lambda} \\
(\AA)\end{array}$ & $\begin{array}{c}\text { Flux } \\
\left(\mathrm{Wm}^{-2} \mu \mathrm{m}^{-1}\right) \\
\left(10^{-17}\right)\end{array}$ & Comment \\
\hline 0.6300 & O I & -1.1 & 0.02 & - \\
\hline 0.6563 & $\mathrm{H} \alpha$ & $-39 /+1$ & 0.89 & P Cygni ${ }^{a}$ \\
\hline 0.7773 & O I & +5 & - & - \\
\hline 0.8498 & Ca II & -7 & 1.26 & - \\
\hline 0.8542 & Ca II & -8 & 1.60 & - \\
\hline 0.8662 & Ca II & -6 & 1.35 & - \\
\hline 1.0830 & He I & $-2 /+7$ & 1.55 & P Cygni ${ }^{b}$ \\
\hline 1.2818 & $\mathrm{~Pa} \beta$ & -6 & 8.91 & $470 \mathrm{~km} \mathrm{~s}^{-1 \mathrm{c}}$ \\
\hline 1.6444 & {$[\mathrm{Fe} \mathrm{II}]$} & -0.3 & 0.91 & very weak \\
\hline 2.0581 & He I & $-1 /+2$ & 3.98 & P Cygni ${ }^{\mathrm{d}}$ \\
\hline 2.1659 & $\mathrm{Br} \gamma$ & -5 & 18.6 & $665 \mathrm{~km} \mathrm{~s}^{-1 \mathrm{c}}$ \\
\hline 2.2940 & $\mathrm{CO}$ & +1 & - & weak bandheads \\
\hline 4.0509 & $\operatorname{Br} \alpha$ & -10 & 35.5 & $415 \mathrm{~km} \mathrm{~s}^{-1 \mathrm{c}}$ \\
\hline 4.6741 & $\mathrm{CO}_{\text {ice }}$ & +57 & - & $830 \mathrm{~km} \mathrm{~s}^{-1 \mathrm{c}}$ \\
\hline
\end{tabular}

a $\mathrm{FWHM}=450 \mathrm{~km} \mathrm{~s}^{-1}$, absorption to $-950 \mathrm{~km} \mathrm{~s}^{-1}$, minimum at $690 \mathrm{~km} \mathrm{~s}^{-1}$, red tail to $+830 \mathrm{~km} \mathrm{~s}^{-1}$.

b $\mathrm{FWHM}=320 \mathrm{~km} \mathrm{~s}^{-1}$, absorption to $-760 \mathrm{~km} \mathrm{~s}^{-1}$, minimum at $300 \mathrm{~km} \mathrm{~s}^{-1}$.

c FWHM of emission component.

d $\mathrm{FWHM}=400 \mathrm{~km} \mathrm{~s}^{-1}$, absorption to $-850 \mathrm{~km} \mathrm{~s}^{-1}$, minimum at $290 \mathrm{~km} \mathrm{~s}^{-1}$.

features are given in Table 2 The molecular CO overtone bandheads longwards of $2.294 \mu \mathrm{m}$ are weakly in absorption in both February 2007, very weakly in absorption in August 2008, while in October 2004 they were strongly in emission. The water vapor absorption bands, at 1.4, 1.9 , and $2.5 \mu \mathrm{m}$, are strongly in absorption, and appear much deeper in August 2008 than in either February 2007 or December 2004. The other broad feature seen in August 2008 is the water ice absorption band, centered at around $3 \mu \mathrm{m}$. It appears somewhat different in shape in August 2008 with respect to the two earlier spectra with a less rounded minimum and a more gradual increase back to the continuum on the long-wavelength side. Finally, the August 2008 spectrum shows the presence of the $4.674 \mu \mathrm{m}$ CO ice absorption band (not included in the plot shown in Fig. 3). This band was previously observed in V1647 Ori by Vacca et al. (2004), Rettig et al. (2005), and Gibb et al. (2006). Rettig et al. modeled the band shape using polar, and apolar CO ice and found that a predominantly apolar $\mathrm{CO}$ matrix ice best fit the February 2004 data with a temperature of $<20 \mathrm{~K}$. Their conclusion was that the line-of-sight CO ice had not been thermally processed and was consistent with that found towards quiescent dark clouds and region of low-mass star formation. A qualitative comparison of the band shape in our August 2008 spectrum with that in the February 2004 spectrum of Vacca et al. (2004) suggests that little has changed in the characteristics of the $\mathrm{CO}$ ice absorption in the intervening four years.

Using the $\mathrm{Pa} \beta$ and $\mathrm{Br} \gamma$ line fluxes, we can estimate mass accretion luminosities $\left(\mathrm{L}_{a c c}\right)$ and rates $\left(\log \left(\mathrm{M}_{\odot} \mathrm{yr}^{-1}\right)\right)$ in a manner similar to that presented in ABR08. Using the relationships defined by Muzerolle et al. (1998) and Gullbring et al. (1998), we find that in August 2008 the line fluxes shown in Table2 resulted in values of $\mathrm{L}_{a c c}=1.42 \mathrm{~L}_{\odot}$ and $\log \left(\mathrm{M}_{\odot} \mathrm{yr}^{-1}\right)=-6.28$ for $\mathrm{Pa} \beta$, and $\mathrm{L}_{a c c}=12 \mathrm{~L}_{\odot}$ and $\log \left(\mathrm{M}_{\odot} \mathrm{yr}^{-1}\right)=-5.34$ for $\operatorname{Br} \gamma$. This assumes a stellar temperature of $\mathrm{T}_{\text {eff }}=3800 \mathrm{~K}$, a stellar luminosity of $\mathrm{L}_{*}=4.9 \mathrm{~L}_{\odot}$, and an inner radius for the accretion disk of $\mathrm{R}_{i n}=5 \mathrm{R}_{*}$ as in ABR08. For the August 2008 fluxes, we also assumed a visual extinction of $\mathrm{A}_{V}=6$ magnitudes. This was determined by dereddening the observed NIR (J-H and H-K) colors of V1647 Ori to typical $\mathrm{T}$ Tauri star values as in ABR08 (see their Figs. 13 and 14). We note that the values of $\mathrm{L}_{a c c}$ and $\log \left(\mathrm{M}_{\odot} \mathrm{yr}^{-1}\right)$ derived from $\mathrm{Pa} \beta$ and $\mathrm{Br} \gamma$ are inconsistent being larger from $\mathrm{Br} \gamma$ by factors of $\sim 8$ and $\sim 9$, respectively. No value of $\mathrm{A}_{V}$ resulted in similar values from the two lines. We can perhaps attribute this difference to the emission in $\mathrm{Pa} \beta$ being optically thick while in $\mathrm{Br} \gamma$ the emission is less so. The ratio of the dereddened $\left(\mathrm{A}_{V}=6\right)$ fluxes, $\mathrm{Pa} \beta / \mathrm{Br} \gamma$, is $\sim 1$ which, from the observations and analysis shown in Fig. 15 from Muzerolle et al. (2001), is a factor $\sim 5 \times$ smaller than that observed in typical 'quiet' classical T Tauri stars. We note that in February 2007, 
when V1647 Ori was supposedly in quiescence, ABR08 derived values of $\sim 4 \mathrm{~L}_{\odot}$ and $\sim-6$ (using the same physical parameters as above with $\left.\mathrm{A}_{V}=19\right)$ from both lines, and a line ratio of $\sim 5$. Clearly, the changes observed in $\mathrm{L}_{a c c}$ and $\log \left(\mathrm{M}_{\odot} \mathrm{yr}^{-1}\right)$ warrant a more detailed investigation.

Our MIR imaging of V1647 Ori showed only a point source and hence, is not explicitly presented here. However, we have extracted photometric values from these data and can compare them to earlier measurements. We find that the integrated flux from V1647 Ori at $\mathrm{N}^{\prime}$ and $\mathrm{Qa}$ is 3.8 and 6.6 Jy, respectively. Similar observations of V1647 Ori in February 2007, when the star had faded to its pre-outburst optical brightness, gave N' and Qa fluxes of 0.23 and $0.44 \mathrm{Jy}$, respectively (ABR08). Earlier measurements quoted by Andrews, Rothberg, \& Simon (2004) showed V1647 Ori to have a $11.3 \mu \mathrm{m}$ flux of $\sim 10 \mathrm{Jy}$. The latter observations were taken soon after the source was discovered by J. McNeil, specifically, in March 2004. Our N' flux of $3.8 \mathrm{Jy}$ is a factor of $2.6 \times$ smaller than the 2004 outburst value and $16.5 \times$ larger than the quiescent phase flux. At $18.6 \mu \mathrm{m}$ (Qa) our flux measurement from September 2008 is $15 \times$ the flux observed in 2007. Thus, the ratio of N/Qa flux in September 2008 is the same (within the associated uncertainities) as in February 2007 at around 0.55×.

\section{SUMMARY}

What we can conclude about the late-August 2008 reappearence of V1647 Ori and McNeil's nebula is that:

In the optical, V1647 Ori and McNeil's Nebula appear photometrically and morphologically similar to when the source was first observed in 2004 i.e. shortly after the eruption occurred.

In the NIR, V1647 Ori appears photometrically similar to February 2004. Spectroscopically, unlike at the start of the 2003 event, $\mathrm{CO}$ overtone emission is not observed. $\mathrm{Br} \gamma$ and $\mathrm{Pa} \beta$ emission are however present. Strong water vapor bands are also seen.

The values of $\mathrm{L}_{a c c}$ and $\log \left(\mathrm{M}_{\odot} \mathrm{yr}^{-1}\right)$ derived from the dereddened $\mathrm{Br} \gamma$ flux (12 $\mathrm{L}_{\odot}$ and -5.3 , respectively) are of a similar order to those found soon after the 2004 outburst (Muzerolle et al. 2005).

Our qualitative interpretation of the results presented above (for the current eruption, the appearance of V1647 Ori in 2007 as described by ABR08, and for the October 2003 eruption as described by AHR09) can be summarized as follows. We believe that the massive accretion event that occurred in 2003 did not actually terminate in 2006 as previously discussed, but only declined in intensity. This is supported by the high-level of accretion found in February 2007 by ABR08, specifically, $\log \left(\mathrm{M}_{\odot} \mathrm{yr}^{-1}\right) \sim-6$. We suggest that the $\sim 6$ magnitude optical fading of V1647 Ori between late 2005 and early 2006 was the result of not only a factor $10 \times$ reduction in accretion luminosity (Muzerolle et al. 2005 derived a value of -5 ), but also the reformation of dust in the immediate circumstellar environment of the star which had been sublimated by the intense radition field from the 2003 accretion burst. We add further support for this speculative interpretation by noting that the change in $\mathrm{A}_{V}$ observed between December 2004 and December 2005 (see Fig. 14 of ABR08) was of a similar order. In early 2008, we consider that a second burst of accretion again sublimated circumstellar dust and resulted in the current $\sim 6$ magnitude brightening of V1647 Ori.

Acknowledgments Based on observations obtained at the Gemini Observatory, which is operated by the Association of Universities for Research in Astronomy, Inc., under a cooperative agreement with the NSF on behalf of the Gemini partnership: the National Science Foundation (United States), the Science and Technology Facilities Council (United Kingdom), the National Research Council (Canada), CONICYT (Chile), the Australian Research Council (Australia), Ministrio da Cincia e Tecnologia (Brazil) and SECYT (Argentina). CA acknowledges the AAS for financial support. BR acknowledges partial support from the NASA Astrobiology Institute under Cooperative Agreement No. NNA04CC08A.

\section{REFERENCES}

Aldering, G., et al. 2002, Proc. SPIE, 4836, 61

Aldering, G., et al. 2006, ApJ, 650, 510

Ambartsumian, V. A. 1971, Astrophysics, 7, 331

Andrews, S. M., Rothberg, B., \& Simon, T. 2004, ApJ, 610, L45

Aspin, C., Greene, T.R., \& Reipurth, B. (2009) AJ, in press (AGR09)

Aspin, C. et al. (2009) AJ, in preparation.

Aspin, C., Herbig, G.H., Reipurth, B. (2009) AJ, in preparation (AHR09)

Aspin, C. 2008, IAU Circ., 8969, 2

Aspin, C., Beck, T. L., \& Reipurth, B. 2008, AJ, 135, 423 (ABR08)

Aspin, C., Barbieri, C., Boschi, F., Di Mille, F., Rampazzi, F., Reipurth, B., \& Tsvetkov, M. 2006, AJ, 132, 1298

Brittain, S., Rettig, T. W., Simon, T., Balsara, D. S., Tilley, D., Gibb, E., \& Hinkle, K. H. 2007, ApJ, 670, L29

Cohen, M., Walker, R. G., Carter, B., Hammersley, P., Kidger, M., \& Noguchi, K. 1999, AJ, 117, 1864

Cushing, M. C., Vacca, W. D., \& Rayner, J. T. 2004, PASP, 116, 362

Davies, R. L., et al. 1997, Proc. SPIE, 2871, 1099

Draper, P.W.,

Taylor, M., \& Allan, A. (2006) Starlink User Note (SUN) 139.17, http://www.starlink.rl.ac.uk/star/docs/sun139.htx/sun139.html
Gibb, E. L., Rettig, T. W., Brittain, S. D., Wasikowski, D., Simon, T., Vacca, W. D., Cushing, M. C., \& Kulesa, C. 2006, ApJ, 641, 383

Gullbring, E., Hartmann, L., Briceno, C., \& Calvet, N. 1998, ApJ, 492, 323

Hartmann, L., \& Kenyon, S. J. 1996, ARA\&A, 34,

Herbig, G. H. 1966, Vistas in Astronomy, 8, 109

Herbig, G. H. 1977, ApJ, 217, 693

Herbig, G. H. 1989, Low Mass Star Formation and Pre-main Sequence Objects, 233

Hook, I. M., Jørgensen, I., Allington-Smith, J. R., Davies, R. L., Metcalfe, N., Murowinski, R. G., \& Crampton, D. 2004, PASP, 116,425

Hunt, L. K., Mannucci, F., Testi, L., Migliorini, S., Stanga,

R. M., Baffa, C., Lisi, F., \& Vanzi, L. 1998, AJ, 115, 2594

Itagaki, K., Nakano, S., \& Yamaoka, H. 2008, IAU Circ., 8968

Lantz, B., et al. 2004, Proc. SPIE, 5249, 146

McGehee, P. M., Smith, J. A., Henden, A. A., Richmond, M. W., Knapp, G. R., Finkbeiner, D. P., Ivezić, Ž., \& Brinkmann, J. 2004, ApJ, 616, 1058

McNeil, J. W. 2004, IAU Circ., 8284

Meixner et al. 2008, Proc. SPIE, 7014, 70142W

Muzerolle, J., Hartmann, L., \& Calvet, N. 1998, AJ, 116, 2965

Muzerolle, J., Calvet, N., \& Hartmann, L. 2001, ApJ, 550, 944 
Muzerolle, J., Megeath, S. T., Flaherty, K. M., Gordon, K. D., Rieke, G. H., Young, E. T., \& Lada, C. J. 2005, ApJ, 620, L107

Rayner, J. T., Toomey, D. W., Onaka, P. M., Denault, A. J.,

Stahlberger, W. E., Vacca, W. D., Cushing, M. C., \& Wang, S. 2003, PASP, 115, 362

Reipurth, B., \& Aspin, C. 2004, ApJ, 606, L119

Reipurth, B., \& Aspin, C. 2004, ApJ, 606, L119
Rettig, T.W., Brittain, S.D., Gibb, E.L., Simon, T., \& Kulesa, C. 2005, ApJ, 626, 245

Telesco, C. M., Pina, R. K., Hanna, K. T., Julian, J. A., Hon, D. B., \& Kisko, T. M. 1998, Proc. SPIE, 3354, 534

Vacca, W. D., Cushing, M. C., \& Simon, T. 2004, ApJ, 609, L29 